\title{
Criminologie
}

\section{Le rapport présentenciel : outil de la prise de décision judiciaire en matière criminelle et pénale}

\section{Gérald Gallant}

Volume 15, numéro 1, 1982

Droit et justice

URI : https://id.erudit.org/iderudit/017149ar

DOI : https://doi.org/10.7202/017149ar

Aller au sommaire du numéro

Éditeur(s)

Les Presses de l'Université de Montréal

ISSN

0316-0041 (imprimé)

1492-1367 (numérique)

Découvrir la revue

Citer cet article

Gallant, G. (1982). Le rapport présentenciel : outil de la prise de décision judiciaire en matière criminelle et pénale. Criminologie, 15(1), 39-49.

https://doi.org/10.7202/017149ar d'utilisation que vous pouvez consulter en ligne. 
Au Canada, l'utilisation du rapport présentenciel comme instrument de prise de décision au niveau de la détermination de la sentence est un phénomène relativement nouveau. Jusqu'à récemment, la fonction de colliger les informations nécessaires au juge pour l'imposition d'une sentence était exclusivement réservée aux procureurs de la défense et au substitut du procureur général. Les législateurs du temps croyaient de façon évidente que les deux parties adversaires pouvaient se préparer adéquatement pour assister la cour dans ses fonctions de détermination de la sentence.

L'évolution de la philosophie pénale davantage orientée vers le caractère juste et humain de la sentence, l'augmentation du nombre de décisions quotidiennes lourdes de conséquences sur l'avenir du contrevenant, l'existence de principes plus exigeants régissant le "sentencing" et l'évolution des sciences humaines sont autant de facteurs ayant contribué à l'introduction du spécialiste du comportement humain en matière d'éclairage présentenciel.

Dombek et Chitrat (1981) établissent les origines du rapport présentenciel aux Etats-Unis. En effet, ils avancent que l'une des premières lois américaines à poser les bases de la probation fut celle du Massachusetts adoptée en 1880. Le législateur, définissant les responsabilités de l'agent de probation, faisait mention « d'un registre complet d'une enquête qui avait pour but de déterminer si l'accusé était apte à se réformer sans être puni ». Ce registre apparaît comme étant l'une des premières versions du rapport présentenciel contemporain.

Au Canada, bien que le législateur fédéral ait tardé à reconnaître le rôle de l'agent de probation comme un tiers apte à recueillir de façon objective des renseignements pertinents pour la cour, l'Ontario s'est avérée l'une des premières provinces à reconnaître ce besoin dans sa "Loi de la probation." (The Probation Act, R.S.O., 1922, c. 103 , s. 3 (1). L'article 3 de cette loi prévoyait que l'agent de probation devait colliger et rapporter à la cour des renseignements tels que les antécédents, l'histoire familiale, les antécédents judiciai-

* Criminologue. Responsable de l'évaluation des programmes à la direction de la probation, ministère de la Justice du Québec. 
res, la nature de l'emploi et tout autre renseignement concernant un accusé, tel que requis par la cour.

C'est en 1969 que fut reconnu officiellement le rapport présentenciel écrit tel qu'on le connaît aujourd'hui et la responsabilité de sa confection par un agent de probation. L'article 662 (1) du Code criminel prévoit la disposition légale suivante :

Lorsqu'un accusé autre qu'une corporation plaide coupable ou est reconnu coupable d'une infraction, un agent de probation doit, s'il est requis de le faire par une cour, préparer et déposer à la cour un rapport écrit concernant l'accusé afin d'aider celle-ci à imposer une sentence ou à décider si l'accusé devrait être libéré en application de l'article 662.1.

Au Québec, c'est à la même période que la Loi de probation et des établissements de détention (1969, ch. 21) est adoptée et l'article 12 précise les devoirs de l'agent de probation en ces termes :

Tout agent de probation doit : a) faire enquête, à la demande du tribunal qui déclare une personne coupable, sur ses antécédents, son caractère et la possibilité de sa réhabilitation ainsi que tout autre sujet qui la concerne et que lui indique le tribunal; b) faire au tribunal un rapport écrit de son enquête pour l'aider à imposer une sentence au contrevenant.

Le texte fédéral étant imprécis quant aux objectifs, à l'utilisation et au contenu du rapport présentenciel et sa petite histoire déjà relativement tumultueuse et controversée à certains égards, il serait peut-être prétencieux à ce stade-ci de son évolution d'en parler comme d'un outil sûr pour la prise de décision en matière criminelle et pénale. Son développement est plutôt relié à l'évolution des services de probation d'une province à l'autre et l'usage que l'on en fait varie selon les coutumes, les mœurs, et les ressources affectées à l'administration de la justice de chaque province. Il est possible de constater des différences marquées quant à son utilisation, sa forme, son contenu et les politiques administratives régissant son application.

En Ontario, par exemple, le ministère des Services correctionnels (1979) rapporte dans son rapport annuel une production de 15056 rapports présentenciels. En 1980, au Québec, les agents de probation ont produit pour leur part 3214 rapports, soit une augmentation de $21 \%$ comparativement à l'année précédente. Ces différences importantes au niveau de la production quantitative d'une province à l'autre sont attribuables à des facteurs tels que la nature de la criminalité pour laquelle ils sont produits, la forme adoptée, l'analyse et les recommandations effectuées, les ressources affectées à sa production, etc. 
Hogart (1971) a constaté en l'occurrence que des rapports présentenciels étaient demandés par la cour dans $50 \%$ de tous les cas étudiés dans son échantillon des magistrats de l'Ontario. Une étude effectuée par Hagan et O'Donnell en 1978 à Edmonton, en Alberta (Howden, 1980), précise que des rapports présentenciels étaient demandés dans $25 \%$ des cas sentencés au cours de la période couverte par cette recherche. Au Québec, une récente compilation indique que des rapports présentenciels sont produits dans un peu plus de 3\% des causes inscrites au rôle de la cour des Sessions de la paix.

En ce qui a trait à la forme de rapport produit, certaines provinces utilisent une forme standardisée présentée en blocs d'information, comme au Manitoba par exemple, tandis que d'autres utilisent une forme permettant une plus grande liberté d'expression tout en conservant une certaine uniformisation. C'est le cas du Québec. Certaines formes dites " sélectives 》 ou " abrégées 》 ont augmenté en popularité. En Ontario, la pratique du "stand down report " est courante et répandue. Brièvement, il s'agit d'un court rapport présentenciel, soit écrit, soit oral, qui peut être préparé à la cour la même journée. Ce type de rapport est généralement complété en moins de deux heures. Des citoyens bénévoles participent également à la préparation et à la rédaction du rapport présentenciel sous la supervision de professionnels (Dombek et Chitrat, 1981).

En ce qui concerne la possibilité pour l'agent de probation d'effectuer une recommandation en conclusion du rapport des différences marquées sont également observables. En Alberta, le guide administratif concernant l'enquête et la rédaction du rapport présentenciel limite le pouvoir de recommandation des agents de probation à la possibilité de la faire seulement en regard de l'utilisation ou non de la probation. Howden (1980) rapporte que, contraints par une telle politique, les professionnels de cette province ont fait preuve d'une remarquable adaptation en employant un certain nombre de phrases types ayant chacune une signification bien précise.

L'Ontario pour sa part a adopté une politique précise et habile par voie législative concernant cette question. En effet, The Ministry of Correctional Act $(1978, s$. 143) prévoit que l'agent de probation ne peut faire une recommandation au rapport présentenciel qu'à la demande de la cour. Au Québec, la situation concernant cette question est différente. Le Guide pour la préparation du rapport présentenciel (1979) laisse une large discrétion aux agents de probation concernant cette question. Une recherche effectuée sur l'ensem- 
ble des rapports présentenciels produits dans cette province (A. Parizeau 1981) relate que 59,2 des agents de probation de cette province présentent une recommandation précise au terme de leurs rapports. L'auteur ajoute que dans les cas ou aucune recommandation formelle n'apparaît en conclusion du rapport, un « texte bien rédigé contient forcément des appréciations qui sont autant d'indications que l'agent soumet au juge en ce qui a trait à la conduite prévisible que le délinquant va adopter à l'avenir "..

En ce qui concerne l'usage quotidien que l'on fait du rapport présentenciel dans les salles d'audience à la cour, un observateur averti aura vite fait de constater que généralement, il est utilisé de façon judicieuse et devient un outil fort utile à l'éclairage présentenciel. Cependant, cet observateur constatera que tantôt les parties adversaires n'utilisent que certaines bribes tirées hors du contexte global du rapport selon les intérêts à défendre, tantôt on le rejette complètement ou tantôt il n'est aucunement utilisé. Ce même observateur averti pourra également constater que les demandes de rapports présentenciels ne sont pas toujours pleinement justifiées. Dombek et Chitrat (1981) notent que lors d'une conférence régionale de l'Association des juges provinciaux de l'Ontario, plusieurs magistrats se sont accordés à dire que l'avocat de la défense utilise l'agent de probation et le rapport présentenciel comme mesures de substitution à son rôle dans le processus de la détermination de la sentence.

Bref, imprécision, incertitude, confusion, controverse et timidité, tant en ce qui concerne les objectifs que le contenu, caractérisent l'utilisation du rapport présentenciel comme outil de prise de décision judiciaire en matière criminelle. Sur le plan pénal, c'est un outil pratiquement peu ou pas utilisé. Bien qu'à plusieurs égards le rapport présentenciel soit utilisé beaucoup dans certaines provinces et peu dans d'autres, il est difficile de connaître son impact réel sur la détermination de la sentence, la recherche étant pratiquement inexistante concernant cette question et difficile à effectuer en raison des usages multiples que l'on en fait. Certes, le rapport présentenciel au Canada a connu une évolution depuis son apparition à la dernière décennie, mais une évolution que l'on pourrait comparer à un voilier filant à pleine allure sans gouvernail.

Il est sans aucun doute possible que l'éclairage présentenciel puisse mieux desservir les intérêts de l'administration de la justice et ceux du contrevenant. Cependant, des principes fondamentaux doivent être franchement discutés au préalable par toutes les parties 
impliquées dans l'importante fonction de la détermination de la peine et des actions concrètes doivent être entreprises de part et d'autre.

L'esprit de la loi canadienne concernant l'éclairage présentenciel et les objectifs fondamentaux qu'il doit viser est en grande partie responsable des confusions et des controverses qui prévalent en cette matière au Canada. Le législateur est demeuré peu explicite sur la finalité que doit poursuivre le rapport présentenciel. Le texte de loi précise qu'il doit être produit pour la cour «afin d'aider celleci à imposer une sentence ou à décider si l'accusé devrait être libéré en application de l'article 662.1". Pourquoi ce double objectif? Le dernier n'est-il pas compris dans le premier? Le législateur, par souci de précision et désirant respecter le sens qu'il donne à la probation comme sursis de sentence, a-t-il vu la nécessité de ne pas inclure ce concept dans le mot sentence? Ou encore souhaitait-il que le rapport présentenciel se prononce exclusivement et de façon explicite sur la possibilité qu'un contrevenant puisse bénéficier de cette mesure, limitant ainsi le pouvoir discrétionnaire de l'agent de probation en matière de recommandation à la cour?

Pratiquement toutes les administrations provinciales en matière de probation sont victimes de la controverse qui prévaut actuellement à l'endroit de la recommandation au rapport présentenciel. La magistrature ne semble pas être constante dans son attitude. Bien que plusieurs magistrats semblent être en faveur que les agents de probation fassent une recommandation franche, plusieurs sont encore en désaccord. Une étude en Ontario effectuée en 1975 rapporte que «généralement les agents de probation, plus que les juges, souhaitent que le pouvoir de recommandation soit élargi ". Au Québec, il semble exister également des différents parmi la magistrature. Bien qu'une plus grande partie des magistrats semble favorable au principe que l'agent de probation puisse émettre son opinion sans réserve en regard de la mesure pénale apparaissant la plus adéquate pour le contrevenant, certains demeurent réfractaires. Un sondage auprès des juges québécois (Langlois, 1975) ayant principalement pour objectif d'évaluer les appréciations des juges face au travail des agents de probation, révèle que $53 \%$ des magistrats étaient favorables contre 47\%. Dombek et Chitrat (1981) font également état de plusieurs causes en provenance des diverses cours d'appel provinciales illustrant la divergence d'opinion concernant cette question.

Comme déjà illustrée précédemment, cette situation pour le moins confuse n'est pas sans provoquer certains problèmes d'adap- 
tation au niveau des politiques administratives régissant le rapport présentenciel dont les effets sont manifestés au niveau de l'attitude des agents de probation concernant le fait de formuler ou non une recommandation. Gabor et Jayewardene (1978) soulignent qu'avec l'appréhension progressive des juges à accepter une recommandation, il est apparu une tendance chez les agents de probation à s'abstenir de faire des recommandations. L'étude Parizeau (1981) trouve des différences régionales marquées concernant ce dernier point.

Un autre aspect de l'éclairage présentenciel souvent débattu est celui du choix des cas propices à une demande de rapports présentenciels? Brocks (1979) souligne que selon la pratique actuelle certains juges demandent des rapports tandis que d'autres n'en exigent en fait jamais. Une étude menée par Hogarth (1971) relate que 65 magistrats sur 71 demandent un rapport lorsqu'ils considèrent la possibilité d'une probation et que 37 sur 71 l'utilisent lorsqu'ils considèrent une sentence d'incarcération. Encore là, la situation est confuse et le débat n'est pas récent, il est évident qu'en considérant l'affluence des contrevenants pour lesquels la cour doit déterminer une sentence quotidiennement, il serait difficile qu'une demande de rapport présentenciel soit exigée dans chaque cas. Certes, cette pratique aurait pour avantage de favoriser pleinement un des objectifs importants de la détermination de la sentence, celui de l'individualisation de la peine, cependant, pour des raisons évidentes, cette option est difficilement applicable dans l'état actuel des choses. Dans l'immédiat le débat concernant cette question est davantage axé sur les situations où la cour envisage une mesure d'emprisonnement pour les auteurs d'un premier délit et les jeunes contrevenants. En 1969, le Rapport Ouimet préconisait qu'aucune peine d'emprisonnement ne soit infligée à un contrevenant contre lequel ne pèse aucune condamnation antérieure à moins qu'un rapport présentenciel n'ait été au préalable soumis au tribunal. Un traitement similaire devait être réservé aux contrevenants pour qui la cour envisageait une peine d'emprisonnement de plus de six mois.

La Commission de réforme du droit du Canada (1979) est moins nuancée et préconise cette pratique pour toute peine d'emprisonnement. Parker (1977) pour sa part indique qu'un rapport présentenciel devrait être demandé dans les cas suivants : les contrevenants âgés de moins de 21 ans, ceux reconnus coupables d'un délit pour la première fois, les délits d'ordre sérieux et particulièrement ceux de nature sexuelle, dans tous les cas où les juges considèrent la possibilité d'une libération absolue ou conditionnelle. Neil Brook 
(1979) le favorise pour l'option de trois mois d'emprisonnement et les cas de premiers délits. De plus, il est d'avis que dans tous les autres cas, la cour ne devrait pas imposer une sentence sans au moins considérer l'option, et dans la négative en stipuler les raisons dans les notes sténographiques.

Comme on peut le voir, le débat est difficile à trancher et les cours d'appel du Canada n'ont pas développé de critères précis pour orienter l'action des juges dans ce domaine. Cependant, le peu de jurisprudence citée dans la littérature consultée concerne des cas impliquant l'incarcération et des contrevenants auteurs de premier délit. Chose certaine, c'est qu'une discrétion doit être exercée à cet égard et l'on doit éviter de requérir au rapport présentenciel dans les cas où la cour ne dispose réellement pas de tous les renseignements nécessaires pour vérifier l'application des principes objectifs et subjectifs de base régissant la détermination de la peine. Compte tenu des grandes divergences individuelles constatées chez les magistrats en regard de la demande ou de l'utilisation du rapport présentenciel pour déterminer la sentence, les différents organismes, ou auteurs ayant débattu de la question semblent s'entendre pour que l'éclairage présentenciel serve de façon systématique pour les auteurs de premier délit, les contrevenants susceptibles de purger des longs termes d'emprisonnement et les jeunes contrevenants.

Pour leur part, les administrations provinciales en probation font de sérieux efforts dans le but d'encourager la magistrature à utiliser le rapport présentenciel de façon plus rationnelle et mieux structurée en tentant de s'ajuster et s'adapter le mieux possible aux divergences individuelles chez les juges en matière de rapport présentenciel. Les nombreuses formes présentement en usage au Canada en témoignent. La tâche n'est évidemment pas facile puisque d'autres questions font également l'objet de diverses controverses, particulièrement en regard de son contenu.

Les circonstances entourant la commission du délit et la version qu'en fait l'accusé, la liste des antécédents criminels, les références aux antécédents juvéniles, la citation des causes pendantes sont autant de questions ne faisant pas l'objet d'un consensus chez les usagers du rapport présentenciel.

Considérant l'ensemble de la situation qui prévaut en matière d'éclairage présentenciel au Canada, il convient de se demander sérieusement comment il peut réellement devenir un instrument efficace de la prise de décision judiciaire en matière criminelle et pénale. 
Il existe plusieurs motifs pouvant justifier un usage plus fréquent et régulier du rapport présentenciel. Entre autres, Brooks (1979) mentionne les suivantes : les présentations des procureurs au moment de la détermination de la sentence sont souvent de courte durée et à caractère peu informatif. Les faits sont souvent peu connus et sont généralement obtenus de la part des accusés. 11 n'existe donc pas d'opportunité pour la cour de les vérifier, tandis que s'ils sont présentés par un tiers, cela facilite l'entente entre les procureurs. Cela évite également que certains faits pertinents soient oubliés ou cachés. Dans sa forme actuelle, le rapport présentenciel donne beaucoup d'information personnelle concernant le contrevenant, sans obliger l'accusé à renseigner la cour publiquement. Dans plusieurs cas, le rapport fait valoir le point de vue de la victime sans qu'elle soit obligée d'être présente à la cour ou encore fournit des informations utiles concernant les ressources disponibles dans la communauté. Enfin, le rapport présentenciel augmente les probabilités que la décision du tribunal soit plus conforme aux principes et aux objectifs du "sentencing ".

Bien que son utilisation soit davantage répandue que lors de sa création, il est cependant pertinent à ce stade-ci de son évolution de se demander si l'instrument créé par des juristes pour des juristes, pour les aider à s'acquitter de l'importante responsabilité de la détermination de la peine, n'est pas encore teinté de préoccupations relevant avant tout de la nature humaine des gens qui en sont responsables et des tracasseries d'ordre légal, plutôt qu'axé véritablement sur la clientèle fréquentant les cours. À vouloir rendre une justice trop juste, il est facile de se perdre dans des détails purement d'ordre technique et légal et d'oublier la véritable finalité des instruments ou des moyens que l'on s'est donnés. À cela s'ajoute le fait que l'introduction du spécialiste des sciences humaines dans une fonction traditionnellement et exclusivement occupée par des gens ayant une formation légale ne semble pas encore une situation acceptée d'emblée. Nombre de textes consultés font mention de cette crainte que les spécialistes du comportement humain ne se substituent au rôle de la cour au niveau de la détermination de la peine.

D'autre part, les spécialistes du comportement humain ont également leur part de responsabilité à assumer en ce qui concerne la situation actuelle du rapport présentenciel. Dans certains cas, la valeur intrinsèque des évaluations et des recommandations soumises est contestable. Dans d'autres cas, la lecture de certains rapports donne vraiment l'impression que l'auteur se substitue au rôle du ju- 
ge. Plusieurs recherches effectuées ont mis en évidence l'importance de la présentation d'un rapport, le type de raisonnement utilisé, le choix du vocabulaire, sa longueur, etc. Cependant, plusieurs sondages effectués de part et d'autre au Canada auprès de la magistrature indiquent que le taux de satisfaction est généralement élevé.

Afin qu'il devienne un instrument efficace de prise de décision au niveau du tribunal, il est d'abord primordial de s'entendre sur sa véritable finalité à savoir un instrument pouvant fournir à la cour de la façon la plus objective possible des renseignements exacts et pertinents pour qu'elle puisse choisir parmi l'éventail varié des mesures pénales, présentement à la disposition du tribunal, la plus appropriée pour l'accusé.

D'autre part, selon Gabor et Jayewardene (1979), l'instrument ne devrait pas ressembler à un catalogue de faits isolés et manquant de cohésion. Il devrait plutôt consister en une série de renseignements disponibles et vérifiables ayant pour but d'évaluer le potentiel de réhabilitation du contrevenant, sa propension à récidiver et le degré de dangerosité qu'il pose pour la société. Le tout devrait être pondéré de façon objective pour en arriver aux conclusions et aux recommandations appropriées. Donc, un rapport présentenciel ainsi préparé ne devrait laisser le juge qu'avec très peu ou pas d'option. Outre cette définition présentée par ces auteurs, un plan d'intervention détaillé et bien appuyé devrait apparaître dans tous les rapports.

De son côté, la cour devrait considérer un rapport ainsi confectionné comme une expertise ou une évaluation présentencielle portant spécifiquement sur ces points au même titre que toute autre expertise demandée en matière médicale, psychiatrique ou autre. Brooks (1979) mentionne que jusqu'à récemment, la majorité des juges et des agents de probation croyaient que le rapport présentenciel le plus utile devrait présenter un portrait complet de l'accusé et être confectionné à partir du plus grand nombre possible de sources d'information.

De fait peu d'information contenue dans un rapport présentenciel traditionnel semble reliée au choix de la mesure la plus appropriée parmi l'éventail varié des sentences disponibles. Un exemple courant est celui de l'histoire sociale détaillée outre mesure dans beaucoup de rapports. Certains commentateurs ajouteront que l'exclusion de l'histoire sociale desservirait les autres usages que l'on fait du rapport présentenciel en l'occurrence comme un document utile de supervision en probation, une source de renseignements va- 
lables pour les institutions carcérales ou autres, ou encore pour fins de recherche universitaire. À notre avis, la finalité première de l'évaluation présentencielle étant d'éclairer le mieux possible la cour pour qu'elle puisse rendre une sentence adéquate, c'est exclusivement cet objectif que le rapport doit poursuivre et dont son contenu doit tẻmoigner.

Certaines études connues : celle de Carter et Wilkins (1976), Wilkins et Chandler (1965), Carter (1967), abondamment citées dans la littérature consultée tendent à démontrer que l'agent de probation arrive assez rapidement à une prise de décision sans pour autant multiplier les sources d'information. Les facteurs influençant le plus sa recommandation seraient la personnalité et le caractère du contrevenant, sa situation familiale actuelle, sa stabilité générale et ses antécédents judiciaires. Gabor et Jayewardene (1978) ajoutent que trop d'information a pour effet de rendre le processus de décision lourd et complexe. Un message trop long aurait pour effet de fatiguer le preneur de décision tandis qu'un message trop court pourrait être perçu comme ayant peu de crédibilité. La conférence de cas est également une pratique répandue chez les agents de probation dans le but de diminuer l'infiltration de biais subjectifs au niveau de la recommandation.

Enfin, les diverses administrations des services de probation au Canada ont également leur part de responsabilité si l'on désire donner au rapport présentenciel son véritable sens d'un instrument efficace de prise de décision judiciaire en matière criminelle et pénale. D'une part, ils ont la responsabilité de pourvoir une formation adéquate et véritable en matière de confection de rapport présentenciel. Malheureusement, les sommes d'argent réservées à la formation du personnel sont généralement négligeables et parfois dépensées en fonction des intérêts personnels des professionnels plutôt qu'en fonction des besoins prioritaires de la clientèle qu'ils desservent. Une supervision adéquate de la qualité et de la valeur des évaluations présentencielles acheminée dans les cours est également une responsabilité importante. Comme le suggère Howden (1980), les diverses administrations auraient sans doute avantage à développer des outils pour mieux structurer la prise de décision de l'agent de probation en matière de recommandation.

La responsabilité de voir à ce que le rapport présentenciel desserve mieux les intérêts de la clientèle judiciaire demeure avant tout une responsabilité collective de tous ceux qui sont impliqués dans le processus de la détermination de la peine. Dans beaucoup de juri- 
dictions, l'éclairage présentenciel tel qu'on le connaît aujourd'hui a maintenant franchi le cap de la décennie. Il appartient à tous les juges, procureurs, agents de probation et administrateurs de faire un examen de conscience sérieux à son égard, de discuter franchement et ouvertement, dans un climat de collaboration et avec le souci de mieux rendre justice, les diverses controverses dont il fait présentement l'objet et, finalement, d'assumer pleinement et entièrement l'important rôle qui leur est confié au niveau de la détermination de la peine. C'est le défi à relever au cours de la prochaine décennie.

\section{BIBLIOGRAPHIE}

BROOKS, N. (1979) : Procedure and Evidence at Sentencing Inquiries (texte inédit), Ottawa, Law Reform Commission of Canada.

CANADA. COMITÉ CANADIEN DE LA RÉFORME PENALE ET CORRECTIONNELLE (1969) : Rapport du Comité canadien de la réforme pénale et correctionnelle, Justice pénale et correction : un lien à forger (Rapport Ouimet), Ottawa, Information Canada.

CANADA. COMMISSION DE REFORME DU DROIT (1979) : Principes directeurs : sentences et mesures non sentencielles dans le processus pénal, Ottawa, Commission de réforme du droit du Canada.

DOMBECK, C.F., CHITRAT, M.W. (1981) : «The Pre-Sentence Report: An Update », The Criminal Law Quarterly, vol. 23, no 2, p. 216.

GABOR, T., JAYEWARDENE, C.H.S. (1978) : "The Pre-Sentence Report as a Persuasive Communication ", Canadian Journal of Criminology, vol. 20, no 1, p. 18.

HOGARTH, J. (1971) : Sentencing as a Human Process, Toronto, University of Toronto Press.

HOWDEN, D. (1980) : «Structuring Decision-making in Probation 》, Le Forum canadien de criminologie, vol. 3, no 1, p. 15.

LANGLOIS, R. (1975) : Sondage auprès des juges de la province de Québec concernant les rapports présentenciels préparés par les agents du Service de probation, Québec, Service de probation, ministère de la Justice.

MINISTĖRE DE LA JUSTICE DU QUÉBEC, Direction de la probation (1979) : Guide pour la préparation du rapport présentenciel.

MINISTRY OF CORRECTIONAL SERVICES (1979) : Annual Report of the Minister for the year ending March 31, Ontario, Ministry of Correctional Services.

PARIZEAU, A. (1981) : Recherche sur le rapport présentenciel, Montréal, Université de Montréal, Centre international de criminologie comparée.

PARKER, G. (1977) : "The Law of Probation ", Canadian Journal of Criminology and Correction. p. 99.

PROBATION OFFICERS ASSOCIATION (1975) : The Report of the Presentence Report Study, Ontario, Probation Officers Association. 